\title{
Gesundheitswesen Schweiz
}

\author{
Die Rolle der FMH in der Gestaltung \\ der Gesundheitsversorgung
}

Als Verband der in der Schweiz berufstätigen Ärztinnen und Ärzte ${ }^{1}$ nimmt die FMH durch ihre Standesordnung ihre Verantwortung für zeitgemässe berufsethische Regeln wahr und stellt durch das SIWF die hochstehende Weiter- und Fortbildung der Ärzteschaft sicher. Ihr Einsatz gilt der Berufsausübung nach den geltenden Regeln der ärztlichen Kunst, zum Wohle der Patientinnen und Patienten und unter Berücksichtigung der ethisch legitimen Interessen der Gesellschaft.

Die FMH vertritt die Interessen der Ärzteschaft, indem sie diese in ihrer Berufstätigkeit in jeglicher Hinsicht unterstützt und sich für Rahmenbedingungen einsetzt, die eine für Patienten und Ärztinnen optimale Berufsausübung erlauben. Die FMH leistet einen Beitrag zur erfolgreichen Bewältigung der wichtigsten Herausforderungen in der Gesundheitsversorgung, indem sie als verlässlicher und glaubwürdiger Partner die Zusammenarbeit zwischen den Akteuren des Gesundheitssystems vorantreibt. Im Sinne einer guten Zusammenarbeit zwischen Gesundheitsfachpersonen, Politik und Administration engagiert sich die FMH für eine ausreichende Einbindung der Ärzteschaft in politische Entscheidungsprozesse sowie für ihre Vertretung in der Exekutive auf nationaler und kantonaler Ebene.

\section{Standortbestimmung und Herausforderungen}

Die Schweizer Bevölkerung erfreut sich einer sehr hohen Lebenserwartung und -qualität sowie einer sehr guten Gesundheit. Das Gesundheitssystem der Schweiz erweist sich in internationalen Vergleichen als eines der besten mit einem sehr guten Zugang zu einem breiten, qualitativ hochstehenden Leistungsangebot und mit kaufkraftbereinigt vergleichsweise moderaten volkswirtschaftlichen Kosten. Auch die Zufriedenheit kranker Menschen mit dem Gesundheitssystem ist in der Schweiz am höchsten. Um den hohen Standard der Gesundheitsversorgung zu erhalten und weiterzuentwickeln, trägt die FMH zu kontinuierlichen Verbesserungen bei. Die notwendigen fortlaufenden Anpassungen in unserem Gesundheitswesen müssen gegenwärtig vor allem den Veränderungen in Demographie und Morbiditätsspektrum sowie dem technischen Fortschritt und den begrenzten Ressourcen Rechnung tragen. Das Hauptkriterium für sinnvolle Reformen muss immer der Mehrwert für die Patientinnen und
Patienten im Rahmen einer gesellschaftlichen und volkswirtschaftlichen Gesamtbeurteilung sein.

\section{Prioritäten und Handlungsfelder für ein nachhaltiges und zukunftsweisendes Gesundheitssystem}

\section{Patientinnen und Patienten im Mittelpunkt}

Den entscheidenden Massstab ärztlichen Handelns bilden das gesundheitliche Wohl und das Selbstbestimmungsrecht der Patientin. Die FMH setzt sich für ein patientenbezogenes Gesundheitswesen ein, das zum Beispiel die freie Arztwahl ermöglicht, die Selbstbestimmung der Patientinnen sowie die ärztliche Schweigepflicht hoch achtet und die Beziehung zwischen Patient und Ärztin sowie die Behandlungsentscheidungen nicht durch ökonomische Kriterien beeinträchtigt.

\section{Interdisziplinarität und Interprofessiona-} lität: Förderung innovativer und integrativer medizinischer Versorgungsmodelle

Durch die zunehmende Spezialisierung und die grösser werdende Zahl an Gesundheitsfachpersonen, die an der Versorgung eines Patienten beteiligt sind, bildet die ganzheitliche Behandlung von Patientinnen heute mehr denn je eine Herausforderung. Gleichzeitig erfordern sowohl die demographischen und Morbiditätstrends in der Bevölkerung als auch der ärztliche Nachwuchsmangel und die vermehrte Teilzeitarbeit innovative und integrative Versorgungsmodelle.

Die FMH fördert darum Entwicklungen, die der Verbesserung der Zusammenarbeit und der Überwindung der Schnittstellen bei der Behandlung dienlich und dem Patientenwohl zuträglich sind. Da die grosse Mehrheit gesundheitlicher Probleme durch die ambulante ärztliche Grundversorgung abschliessend behandelt werden kann, sind hierbei die Stärkung und Förderung der Haus- und Kinderarztmedizin unter optimaler Vernetzung und Koordination mit anderen Disziplinen und Professionen von Bedeutung.

\section{Qualitätssicherung auf Basis ärztlicher Expertise}

Das Sicherstellen der Qualität ist von jeher ein wesentlicher Bestandteil der ärztlichen Tätigkeit. Die FHM nimmt in der Förderung und Weiterentwicklung der Behandlungsqualität eine Vorreiterrolle ein. Sie fördert die Zusammenarbeit innerhalb der Ärzteschaft und die Vernetzung von Qualitätsaktivitäten, koordiniert Qua- 
litätsfragen auf nationaler Ebene und bezieht die Partner im Gesundheitswesen ein. Mit der Gründung der Schweizerischen Akademie für Qualität in der Medizin SAQM hat die FMH ärztliche Qualitätsaktivitäten institutionell verankert und trägt zur Nutzung von Synergien und zur Vermeidung von Doppelspurigkeiten bei.

\section{Für eine bedarfsgerechte und zukunfts- weisende Aus-, Weiter- und Fortbildung}

Eine qualitativ hochstehende und auf den Bedarf an präventiven, kurativen und palliativen Leistungen ausgerichtete ärztliche Bildung ist ein zentrales Anliegen der Ärzteschaft. Die FMH hat zu diesem Zweck 2009 das Schweizerische Institut für ärztliche Weiter- und Fortbildung SIWF gegründet, das unter anderem die eidgenössischen Facharzttitel regelt und erteilt. FMH und SIWF setzen sich für ausreichend Studienplätze in der Medizin, für eine hohe Qualität der Weiter- und Fortbildung, für eine adäquate Finanzierung der Weiterbildung und für bildungsfreundliche Arbeitsbedingungen ein.

\section{Gesundheitsförderung und Prävention: zentrale ärztliche Aufgaben im Dienste} von Patientinnen und Bevölkerung

Gesundheitsförderung und Prävention bilden in der ärztlichen Tätigkeit ein Handlungsfeld von zunehmender Bedeutung. Angesichts vermehrt lebensstilbezogener Gesundheitsprobleme, aber auch in Bezug auf Infektionserkrankungen oder weitere Gesundheitsprobleme, wie psychische Belastungen, Suchtverhalten und nicht-übertragbare Krankheiten, kommen zudem Public-Health-Ansätzen auf Bevölkerungsebene vermehrt Bedeutung zu.

\section{Weiterentwicklung von eHealth und Einsatz für den Datenschutz}

Die Weiterentwicklung und flächendeckende Implementierung von eHealth können einen Beitrag zu effizienterem Arbeiten im Gesundheitswesen leisten, die interdisziplinäre wie interprofessionelle Zusammenarbeit vereinfachen sowie die Patientensicherheit erhöhen. Die

Korrespondenz:

Dr. med. Jürg Schlup Präsident der FMH

Elfenstrasse 18

Postfach 300

CH-3000 Bern 15

Informationen zur

Entstehung und Abstützung des Papiers sind nachzulesen in der Schweizerischen

Ärztezeitung Nr. 40/2016,

S. 1366: Entstehungsprozess

und Resultat.

Online: http://www.saez.ch/ docs/saez/2016/40/de/

saez-05004.pdf
FMH engagiert sich für die Ausgestaltung der eHealthInstrumente mit einem Mehrwert für Patientinnen und Ärzteschaft und arbeitet an den Werkzeugen zur praktischen Umsetzung mit. Die Wahrung des Datenschutzes und das Verhindern einer "gläsernen Patientin" geniessen dabei oberste Priorität. Chancen und Risiken sowie Kosten und Nutzen einzelner eHealth-Instrumente werden transparent aufgezeigt und abgewogen.

\section{Vergütung medizinischer Leistungen: Tarife und Verträge}

Die FMH setzt sich für eine adäquate und nachhaltige Tarifpolitik für die Ärzteschaft im ambulanten wie im stationären Bereich ein. Sie nimmt eine führende Rolle bei der Weiterentwicklung von ambulanten und stationären Tarifsystemen ein und analysiert gesundheitsökonomische Entwicklungen in einer gesamtvolkswirtschaftlichen und sektoriell übergreifenden Gesamtoptik. Sie erfragt die Bedürfnisse der Ärzteschaft und verschafft deren Forderungen in den nationalen Gremien Gehör

Leistungsgerechte, betriebswirtschaftlich korrekte und praktikable Abgeltungssysteme bilden die Grundlage für Wettbewerb und müssen geeignete Rahmenbedingungen für eine optimale Versorgungsqualität und eine hohe Effizienz der Leistungserbringung schaffen. Vergütungssysteme müssen gleiche Preise für vergleichbare Leistungen vorsehen und dürfen nicht einzelne Fachdisziplinen begünstigen oder benachteiligen.

Unter Berücksichtigung von Wirksamkeit, Zweckmässigkeit und Wirtschaftlichkeit (WZW) darf die therapeutische Freiheit keinesfalls durch Vergütungssysteme beschnitten werden. Weiterhin muss die Unabhängigkeit gegenüber der Industrie gewahrt bleiben. Die Leistungserfassung muss von hoher Transparenz und möglichst geringem administrativen Aufwand gekennzeichnet sein

\section{Arbeitsbedingungen der Ärzteschaft}

Ein gutes und patientenorientiertes Gesundheitswesen setzt optimale Rahmenbedingungen voraus, die Ärztinnen und Ärzten ermöglichen, qualitativ hochstehende Leistungen zu erbringen. Kernvoraussetzungen bilden dabei Aspekte wie die Gewährleistung der Therapiefreiheit, faire Arbeitsbedingungen, das Sicherstellen eines leistungsgerechten Einkommens sowie die nötigen Instrumente (z.B. Labor, Röntgen), um den Beruf sinnvoll ausüben zu können. Darüber hinaus fordert die FMH familienfreundliche Arbeitsbedingungen und Arbeitsformen, die den aktuellen Lebensentwürfen entsprechen.

\section{Legitimation und Grenzen staatlicher Regulierung}

Auch wenn die spezifischen Charakteristika des Gesundheitswesens eine im Vergleich zu anderen Märkten stärkere Regulierung erfordern, gilt es auch in diesem Bereich die Möglichkeiten des Wettbewerbs und sinnvoller ökonomischer Anreize auszuschöpfen. Die FMH erachtet die Kompetenzordnung der Bundesverfassung als sinnvoll, die eine Gesetzgebung des Bundes nur für die in der Verfassung genannten Bereiche des Gesundheitswesens vorsieht. Mehr staatliche Planung, Steuerung und Interventionen sind für die Bewältigung der aktuellen Herausforderungen und eine Dämpfung des Kostenwachstums hingegen nicht zielführend. 